\title{
Propiedades de los suelos en diferentes usos agropecuarios, Las Tunas, Cuba
}

\author{
Properties of soils in different agricultural uses, Las Tunas, Cuba
}

\section{Santa Laura Leyva R. ; Aime Baldoquín P. ${ }^{2}$; Manuel Reyes $0 .^{3}$}

$1 \quad$ Ph.D. Universidad de Las Tunas, Las Tunas, Cuba, laural@ult.edu.cu.

2 M.Sc. Ciencias Agrícolas, Universidad de Las Tunas, Las Tunas, Cuba, abaldoquinp@ult.edu.cu.

3 Ingeniero Agrónomo, Universidad de Las Tunas, Las Tunas, Cuba, manuelro@ult.edu.cu.

Citar: Leyva, S., Baldoquin, A. Reyes, M. (2018). Propiedades de los suelos en diferentes usos agropecuarios, Las Tunas, Cuba. Rev. Cienc. Agr. 35(1):36-47. doi: http://dx.doi.org/10.22267/rcia.183501.81.

Recibido: Octubre 25 de $2016 . \quad$ Aceptado: Septiembre 04 de 2017.

\section{RESUMEN}

La degradación de los pastizales, constituye uno de los problemas más graves relacionados con el uso de los suelos en la zona norte del municipio de Las Tunas, Cuba. El manejo inadecuado de los suelos, ha provocado su deterioro progresivo con impactos negativos en la producción ganadera, lo que ha incidido en la producción de carne y leche, incrementa los costos de producción y causa efectos desfavorables en el medio ambiente. En el presente trabajo se evaluó el efecto de diferentes sistemas de uso agropecuarios en algunas propiedades de los suelos Luvisoles haplicos, desarrollados sobre granitoides. Los sistemas de uso incluyen pastos naturales, pastos cultivados, sistema silvopastoril y un sistema de referencia natural. Las propiedades de suelos medidas fueron la textura, la densidad aparente, la capacidad de campo, la porosidad total y de aireación, velocidad de infiltración, el contenido de materia orgánica y la enzima $\beta$-glucosidasa. Los resultados mostraron que las tecnologías de manejo aplicadas en los pastizales afectan las propiedades de los suelos e inciden en su degradación, con incrementos en la densidad aparente, menores valores de infiltración, porosidad y menor actividad de la enzima ß-glucosidasa lo que limita el funcionamiento del sistema suelo-planta-animal. Se evidenció un efecto conservacionista de los suelos en el sistema silvopastoril, respecto a las propiedades físicas, químicas y biológicas, lo que constituye una alternativa para la recuperación de los suelos de pastizales en los sistemas ganaderos de la región.

Palabras clave: Sistema silvopatoril, compactación, $\beta$-glucosidasa, materia orgánica, pastizales.

\begin{abstract}
The degradation of pastures, constitutes one of the most serious problems related to the use of soils in the northern area of Las Tunas municipality, Cuba. The inadequate management of soils, has led to their progressive deterioration with negative impacts on livestock, which has affected the production of meat and
\end{abstract}


milk, increases of production costs and adverse effects on the environment. In the present work, the effect of different agricultural systems on some properties of haplic Luvisoles soils, developed on granitoids, was evaluated. The systems included natural pastures, cultivated pastures, silvopastoral system and a natural system. The soil properties evaluated were texture, bulk density, field capacity, total porosity and aeration, infiltration speed, organic matter and $\beta$-glucosidase enzyme. The management technologies applied in the grasslands affect the soil properties and their degradation, with increases in the apparent density, lower values of infiltration, porosity and lower activity of the enzyme ß-glucosidase, which limits the functioning of the soil-plant-animal system. It was evident a preserving effect of the soils in the silvopastoral system regarding to the physical, the chemical and the biological properties, which indicates that they are alternatives for the recovery of grassland soils in the cattle systems.

Key words: Silvopastoral system, compaction, $\beta$-glucosidase, organic matter, pasture.

\section{INTRODUCCIÓN}

La degradación de los suelos se ha propagado en el mundo a un ritmo acelerado en los últimos 50 años y especialmente, la degradación del carbono orgánico, ha conllevado a importantes pérdidas en la calidad del suelo y representa una amenaza para los sistemas de producción agrícola y seguridad alimentaria (Verhulst et al., 2015). El suelo se degrada cuando sus propiedades no tienen la posibilidad de regenerarse naturalmente, por lo tanto, debe generar conocimiento adecuado sobre el uso del suelo a través del estudio de sus propiedades, para poder disponer de alternativas en las decisiones sobre la práctica a realizar.

El gran desafío en la actualidad es el diagnóstico de la calidad de vida de los suelos, para poder desarrollar un sistema productivo basado en una agricultura sostenible en sí misma, conservadora de los recursos, eficiente en energía y socialmente viable. Los resultados obtenidos por Hernández et al. (2006) demuestran que con los cambios globales en los suelos, principalmente por el diferente uso de la tierra, cambian las propiedades del suelo y en dependencia del estado de dichas propiedades, será la respuesta del suelo a un cultivo determinado.

Los pastizales constituyen un recurso renovable, y su mantenimiento y mejora puede ser posible si se emplean prácticas culturales que beneficien sus procesos y funciones (Lok, 2016). Por ello, en los diseños de alternativas tecnológicas para los sistemas de pastoreo en el trópico, se insertan en forma rentable y sostenible la adopción de sistemas silvopastoriles intensivos que se soportan en principios agroecológicos (Murgueitio et al., 2016), la introducción de germoplasmas forrajeros, entre ellos el Pennisetum purpureum, debido a su alta producción de biomasa y su adaptación a una gran diversidad de suelos y condiciones climáticas adversas (García et al., 2014), que generan diferentes beneficios, tanto en la producción animal como en la conservación del suelo (Chará et al., 2015).

En sistemas agrosilvopastoriles (Silva et al., 2011) la calidad física de los suelos Luvisoles, adquirió niveles similares a los suelos con vegetación natural. Los contenidos de carbono almacenado en el suelo (CAS) en el sistema silvopastoril y el de mezcla de leguminosas rastreras, tuvieron incremento paulatino en el CAS con el tiempo de explotación, así que los convierte en alternativas viables para la captura de carbono en agroecosistemas de pastizales, mientras el sistema de monocultivo mostró signos de deterioro, con los que se relacionaron sus valores inferiores en el CAS (Lok, 2013).

En la zona norte del municipio de Las Tunas, se encuentra el cinturón granodiorítico, que ocupa el 52,4\% de los suelos del municipio y están destinados a la explotación agropecuaria. En este cinturón, existe una amplia gama de actividades agropecuarias, forestal, cultivos varios, sin cultivar, 
pero predominan las áreas de pastos naturales. La introducción de forrajes y sistemas silvopatoriles, entre otras prácticas, son aún insuficientes en las unidades agropecuarias.

Esta región es considerada de singular importancia por cuanto ocupa una extensión de 47610ha. Los suelos Luvisoles haplicos ocupan la mayor extensión y representan el 51,9\% del cinturón. El mal manejo de los suelos ha causado un impacto negativo sobre los recursos naturales, especialmente el suelo, el agua y la biodiversidad. Debido a lo anterior, se ha reducido la respuesta productiva de los diversos sistemas ganaderos en la región, de ahí que examinar las propiedades de los suelos a través de indicadores permitirá evaluar los efectos de los diferentes sistemas de uso en la calidad de los suelos y a su vez, obtener información que permita tomar decisiones de manejo, con el propósito de garantizar la producción de bienes y proteger los recursos naturales, lo que constituye el objetivo del presente trabajo.

\section{MATERIALES Y MÉTODOS}

El estudio se desarrolló en la Unidad agropecuaria "La Veguita" Vaquería 12, la cual cuenta con una extensión territorial de 62,78ha y se enmarca dentro de las coordenadas geográficas $20^{\circ} 59^{\prime} 40^{\prime \prime} \mathrm{y}$ $21^{\circ} 51^{\prime} 31^{\prime \prime} \mathrm{LN}$ y los 7654'06” y 7659'15”LO. Esta unidad se encuentra insertada en el sistema nacional de vaquería escuela. Se han identificado zonas con actividad pecuaria, sometidas a elevada presión de uso del suelo mediante diferentes sistemas de manejo. Los sistemas de uso investigados, presentan similares condiciones de formación de los suelos, pero se distinguen en cuanto al propósito de la producción, manejo, vegetación dominante y tiempo de explotación. Se seleccionó un área ocupada por algarro (Albizzia procera , Benth), júcaro( Bucida buceras $L$ ), guásima común (Guazuma ulmifolia, Lam), almácigo (Bursera simaruba (L.), Ayúa (Zanthoxylum matinicense L.), Ateje (Cordia colococa ) como referencia del suelo natural (Arboleda).
Un sistema de pasto natural con especies de Sporobolus indicus, Dichantium annulatum y Paspalum notatum (PN); pasto cultivado con Pennisetum purpureum vc CUBA CT-115 y Cynodon nlemfuensis; sistema silvopastoril con Leucaena leucocephala Lam. en franjas y Panicum maximun vc. Likoni. Se utilizó un diseño Completamente Aleatorizado, con tres repeticiones.

En cada uso de la tierra se estableció una calicata principal (1 × 2 × 2m), ubicada en el centro del área, para la descripción de las características morfológicas, según Manual Metodológico para la Cartografía Detallada y Evaluación Integral de los Suelos (Hernández et al., 1995). Se tuvo en cuenta la localización, vegetación, relieve, material originario, uso actual, condiciones meteorológicas, tecnologías de suelo. Para la clasificación de los suelos, se aplicó la World Reference Base (WRB) (IUSS Grupo de Trabajo WRB, 2007). Se tomaron muestras de los horizontes genéticos para la determinación de las variables físicas: textura (método de la pipeta (NRAG 408,1981), densidad de sólidos (método de los volumétricos NRAG-128, 2009), materia orgánica (método Walkley y Black, 1934). La velocidad de infiltración se realizó en tres puntos en cada uso del suelo (método infiltrómetros de los cuadrantes según Vadiunina y Korchagina, (1986). Para determinar la densidad aparente, se seleccionaron cinco puntos al azar y se establecieron minicalicatas tomando muestras a diferentes profundidades de 0-5, 5-10, 10-20, 20-30,0cm en la época de lluvias, coincidiendo con la humedad a capacidad de campo (método del cilindro NC ISO 11272:2003). La capacidad de campo se determinó por el método de campo según (NC 2010). La actividad de la enzima ß- glucosidasa se determinó en las profundidades de 0-10 y 10-20cm por el método propuesto por Hoffmann y Dedeken (1965) y modificado posteriormente por Strobl y Traunmüller (1996). La porosidad total y de aireación, se determinaron mediante fórmulas según (NC: 2010).

Análisis estadísticos. El análisis estadístico de los datos se realizó mediante el uso del paquete SPSS 
versión 15.0 para Windows. Se determinaron los principales estadígrafos (media aritmética y error estándar de la media). Dado que esencialmente se trata del mismo tipo de suelo con características similares en el perfil de las cuatro condiciones de uso, se supuso una diferencia constante por profundidad de muestreo. La prueba de normalidad de las distribuciones de medias se realizó según Shapiro Wilks. Los datos mostraron una distribución normal y se sometieron a un Análisis de Varianza (ANDEVA). La comparación de medias se realizó con la prueba de Tukey $(\mathrm{p}<0,05)$.

\section{RESULTADOS Y DISCUSIÓN}

Análisis granulométrico. En todos los ecosistemas, la arena supera las demás fracciones, los contenidos de arcilla son superiores al limo, con incremento en el horizonte B (Tabla 1). Hay predominio de la arena fina (65 - 80\%), respecto a la arena gruesa (20 - 35\%). De forma general, los suelos desarrollados sobre granodiorita presentan una textura franca arcillosa con incremento de arcilla en profundidad que los clasifica como lixiviados.

Tabla 1. Clasificación textural y distribución de las partículas del suelo en los diferentes sistemas de uso en Las Tunas, Cuba.

\begin{tabular}{ccccccc}
\hline \multirow{2}{*}{ Sistemas } & $\begin{array}{c}\text { Profundidad } \\
(\mathbf{c m})\end{array}$ & Arena gruesa & Arena fina & Limo & Arcilla & Clasificación \\
\hline \multirow{2}{*}{ Arb } & & $(2000-200 \mu \mathrm{m})$ & $(200-20 \mu \mathrm{m})$ & $(20-2 \mu \mathrm{m})$ & $(<2 \mu \mathrm{m})$ & \\
& $0-5$ & 15,48 & 34,25 & 27,84 & 22,43 & Fr-arcillosa \\
& $5-20$ & 12,28 & 31,75 & 27,44 & 28,53 & Arcillosa gruesa \\
& $20-34$ & 14,96 & 29,53 & 25,46 & 30,05 & Arcillosa gruesa \\
& $34-40$ & 11,81 & 30,2 & 23,37 & 34,62 & Arcillosa gruesa \\
& $40-50$ & 12,72 & 33,36 & 17,51 & 36,41 & Arcillosa gruesa \\
\hline PN & $0-5$ & 8,69 & 39,99 & 26,88 & 24,44 & Franco arcillosa \\
& $5-20$ & 11,80 & 36,61 & 25,66 & 25,93 & Arcillosa gruesa \\
& $20-30$ & 11,86 & 30,59 & 22,63 & 34,92 & Arcillosa gruesa \\
& $30-40$ & 12,01 & 30,00 & 20,20 & 37,79 & Arcillosa gruesa \\
& $40-50$ & 21,07 & 36,63 & 18,21 & 24,09 & Fr-Arc-arenosa \\
\hline PC & $0-4$ & 14,34 & 38,88 & 23,38 & 23,4 & Fr-arcillosa \\
& $4-20$ & 17,11 & 37,5 & 21,23 & 24,16 & Fr-arcillosa \\
& $20-30$ & 12,77 & 40,2 & 20,78 & 26,25 & Fr-arcillosa \\
& $30-40$ & 14,07 & 34,76 & 18,7 & 32,47 & Arcillosa gruesa \\
& $40-50$ & 27,62 & 32,78 & 15,11 & 24,49 & Arcillosa gruesa \\
\hline Silv & $0-5$ & 9,00 & 30,19 & 27,11 & 33,70 & Arcillosa gruesa \\
& $5-20$ & 9,58 & 29,10 & 26,36 & 34,96 & Arcillosa gruesa \\
& $20-28$ & 10,26 & 28,80 & 20,73 & 40,21 & Arcillosa gruesa \\
& $40-40$ & 13,77 & 26,50 & 17,10 & 42,63 & Arcillosa gruesa \\
& 24,22 & 30,68 & 16,79 & 28,31 & Arc-arenoso \\
\hline
\end{tabular}

Arb- Arboleda; PN-Pasto natural; PC-Pasto cultivado; Silvop- Silvopastoril 
Densidad aparente. La densidad aparente hasta los $30 \mathrm{~cm}$ de profundidad, varió desde 1,06 a 1,46 $\mathrm{Mg} \mathrm{m}^{-3}$. En los sistemas arboleda y silvopastoril, se observaron los valores significativamente menores de densidad aparente y en los sistemas de pastizales los mayores de densidad, sobre todo a partir de los $5 \mathrm{~cm}$ de profundidad (Tabla 2).

Tabla 2. Densidad aparente $\left(\mathrm{Mg} \mathrm{m}^{-3}\right)$ en los diferentes sistemas de uso del suelo en Las Tunas, Cuba.

\begin{tabular}{lcccc}
\hline \multicolumn{1}{c}{ Sistemas } & \multicolumn{4}{c}{ Profundidad (cm) } \\
\hline & $\mathbf{0 - 5}$ & $\mathbf{5 - 1 0}$ & $\mathbf{1 0 - 2 0}$ & $\mathbf{2 0 - 3 0}$ \\
Arboleda & $1,06 \mathrm{a}$ & $1,22 \mathrm{a}$ & $1,25 \mathrm{a}$ & $1,34 \mathrm{a}$ \\
Pasto natural & $1,24 \mathrm{~b}$ & $1,36 \mathrm{~b}$ & $1,40 \mathrm{~b}$ & $1,44 \mathrm{~b}$ \\
Pasto cultivado & $1,34 \mathrm{c}$ & $1,38 \mathrm{~b}$ & $1,44 \mathrm{c}$ & $1,46 \mathrm{~b}$ \\
Silvopastoril & $1,12 \mathrm{a}$ & $1,25 \mathrm{a}$ & $1,27 \mathrm{a}$ & $1,35 \mathrm{a}$ \\
Error estándar & 0,033 & 0,021 & 0,024 & 0,017 \\
\hline
\end{tabular}

Letras distintas en cada columna indican diferencias significativas $(\mathrm{p}<0,05)$.

Los resultados en la arboleda concuerdan con Chavarría et al. (2012) quienes señalan que en el sistema boscoso, la poca intervención humana, un mayor número de lombrices y contenido de materia orgánica, explican una menor densidad aparente.

En los sistemas de pastizales a partir de los $10 \mathrm{~cm}$ de profundidad, las densidades aparentes son superiores a $1,4 \mathrm{Mg} \mathrm{m}^{-3}$, umbrales considerados como críticos (Griffith et al., 1977) para el desarrollo de los cultivos. La compactación del suelo limita el acceso de la raíz a la humedad y nutrientes en el subsuelo (Celik y Raper, 2012), es por esto que dichos autores sugieren en su manejo la subsolación en la preparación de suelos. Leyva et al. (2014) reportaron para sistemas de pastos cultivados con alta compactación, disminución de la densidad aparente, con la utilización de la subsolación hasta los $30 \mathrm{~cm}$ de profundidad. Según Raper (2005) los suelos con texturas más arenosas en superficie y arcillosas en el subsuelo son susceptibles a la compactación y cuando se aplican presiones altas por el tráfico de animales o maquinaria se logran valores más altos de densidad aparente (Munkholm, 2011). El uso del suelo, la vegetación y el tipo de suelo son factores que se encuentran interrelacionados y determinan las propiedades físicas y químicas de los suelos (Gaspar y Navas, 2013).

Densidad de sólidos $\left(\rho_{s}\right)$ La densidad de sólidos (Figura 1) osciló entre 2,47 y 2,57 Mg.m³. En todos los sistemas se incrementan con la profundidad, a medida que se encuentran materiales menos meteorizados. Estos valores coinciden con los obtenidos por Porta et al. (2003) para densidades con presencia de cuarzo y feldespatos.

En los perfiles de suelo, conforme disminuye la materia orgánica, se incrementa la densidad de sólidos. Sin embargo, en el pasto cultivado, el mantenimiento de los niveles altos de materia orgánica hasta la profundidad de $20 \mathrm{~cm}$ permitió una densidad menor. La materia orgánica pesa mucho menos que un volumen igual de sólidos minerales, por tanto lo que afecta marcadamente a la densidad de las partículas.

Porosidad total y Porosidad de aireación. La porosidad total (Tabla 3) en los primeros $5 \mathrm{~cm}$ fue superior en los sistemas arboleda y silvopastoril evaluada como alta, con diferencias significativas con los demás sistemas. En profundidad, la porosidad disminuye en todos los sistemas de uso alcanzando los valores más bajos, los sistemas de pastos cultivados y naturales, sin diferir entre ellos. El sistema arboleda y silvopastoril, alcanzan una porosidad media. Murillo et al. (2014) encontraron en áreas ganaderas, donde aplicaron prácticas sostenibles, valores mayores de porosidad (41,20\%), respecto a las áreas testigo (34,20\%), en $30 \mathrm{~cm}$ de profundidad. 


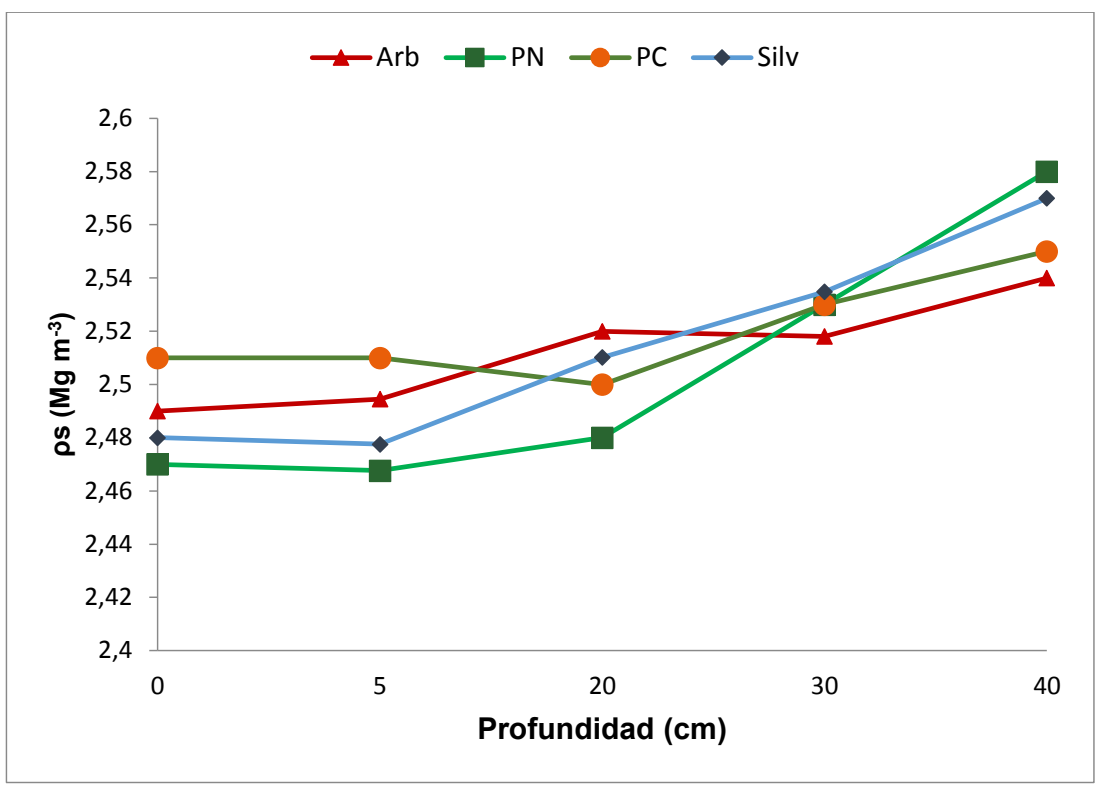

Arb- Arboleda; PN-Pasto natural; PC-Pasto cultivado; Silvop- Silvopastoril

Figura 1. Densidad de sólidos $\left(\mathrm{Mg} \mathrm{m}^{-3}\right)$ en los diferentes sistemas de uso del suelo en Las Tunas, Cuba.

Tabla 3. Porosidad total y de aireación en los diferentes sistemas de uso del suelo en Las Tunas, Cuba.

\begin{tabular}{cccccc}
\hline Sistemas & Prof.(cm) & $\begin{array}{c}\text { Pt } \\
\text { \% }\end{array}$ & $\begin{array}{c}\text { CC } \\
\text { \% peso }\end{array}$ & $\begin{array}{c}\text { CC } \\
\text { \%vol }\end{array}$ & $\begin{array}{c}\text { Pa } \\
\text { \% }\end{array}$ \\
\hline Arboleda & $0-5$ & $57,60 \mathrm{a}$ & 29,0 & 30,74 & $26,86 \mathrm{a}$ \\
& $5-20$ & $\begin{array}{c}50,79 \mathrm{a} \\
27,3\end{array}$ & $\begin{array}{c}33,85 \\
16,94 \mathrm{a}\end{array}$ \\
\hline Pasto & $20-30$ & $46,83 \mathrm{a}$ & 27,8 & 37,25 & $9,57 \mathrm{a}$ \\
Natural & $0-5$ & $49,80 \mathrm{~b}$ & 29,0 & 35,96 & $13,84 \mathrm{c}$ \\
& $5-20$ & $43,95 \mathrm{~b}$ & 28,0 & 38,92 & $5,03 \mathrm{c}$ \\
Pasto & $20-30$ & $43,08 \mathrm{~b}$ & 29,0 & 41,76 & $1,32 \mathrm{c}$ \\
cultivado & $0-5$ & $47,22 \mathrm{~b}$ & 27,7 & 36,84 & $10,38 \mathrm{c}$ \\
& $5-20$ & $43,20 \mathrm{~b}$ & 27,5 & 39,05 & $4,15 \mathrm{c}$ \\
Silvo & $20-30$ & $42,52 \mathrm{~b}$ & 27,3 & 39,86 & $2,66 \mathrm{c}$ \\
pastoril & $0-5$ & $54,84 \mathrm{a}$ & 31,0 & 34,72 & $20,12 \mathrm{~b}$ \\
& $5-20$ & $49,40 \mathrm{a}$ & 30,0 & 38,10 & $11,30 \mathrm{~b}$ \\
Error & $20-30$ & $46,85 \mathrm{a}$ & 30,0 & 40,50 & $6,35 \mathrm{~b}$ \\
\hline Estándar & $0-5$ & 1,13 & & & 1,65 \\
& $5-20$ & 0,91 & & & 1,38 \\
& $20-30$ & 0,73 & & & 1,00 \\
\hline
\end{tabular}

Medias con letras distintas en cada columna indican diferencias significativas $(p<0,05)$. 
Sadeghian et al. (2010) encontraron en potreros valores inferiores de porosidad $(32 \%)$ en $10 \mathrm{~cm}$ de profundidad y lo relacionan con el incremento de la densidad. La porosidad de aireación es un criterio importante para estimar la disponibilidad de oxígeno a las raíces de las plantas. El sistema arboleda alcanzó el mayor valor de porosidad de aireación en todas las profundidades, evaluada como alta en los primeros $5 \mathrm{~cm}$, con diferencias significativas con los demás sistemas, seguido por el sistema silvopastoril con valores significativamente mayores que los demás en todas las profundidades.

En los sistemas de pastizales, la porosidad de aireación en la profundidad de $5-20 \mathrm{~cm}$, fue inferior al 10\%, límite señalado como crítico para el desarrollo de las raíces, que determina la baja productividad de estos sistemas. Es interesante señalar que no solo es importante el análisis de los valores críticos, sino además, los cambios bruscos que se producen en el perfil. Similar distribución vertical de la porosidad de aireación en el perfil han sido obtenidos por Lozano et al. (2000), debido a un incremento de la densidad aparente. Los resultados en arboleda y silvopastoril se han visto favorecidos por ser suelos que presentan una mejor organización estructural y menor compactación.
Velocidad de infiltración. La velocidad de infiltración calculada para los suelos de los diferentes sistemas de uso se muestra en la Figura 2. En el sistema arboleda, se obtuvo una velocidad de infiltración básica mayor que en el resto de los sistemas, moderadamente rápida en el sistema silvopastoril y moderada en los pastos naturales. El sistema de pasto cultivado, tuvo una velocidad de infiltración moderadamente lenta, debido a la alta compactación del suelo, producida por el intenso pastoreo.

Los resultados coinciden con lo señalado por Gómez et al. (2014), quienes encontraron diferencias estadísticas significativas entre la tasa de infiltración de tierras forestales y agrícolas, respecto a la tasa de infiltración de tierras de pastoreo. En los sistemas silvopastoriles, la presencia de las leguminosas de raíces profundas, contribuye a regenerar la estructura del suelo y bioporos, que mejoran el drenaje y la aireación (Kassam, 2014). La cobertura vegetal, el sombrío y las condiciones microclimáticas en estos sistemas, contribuyen a recuperar la macrofauna edáfica, que participa en el proceso de remoción de suelo, evita la compactación y mejora la permeabilidad y la capacidad de retención del agua (Montagnini et al., 2015).

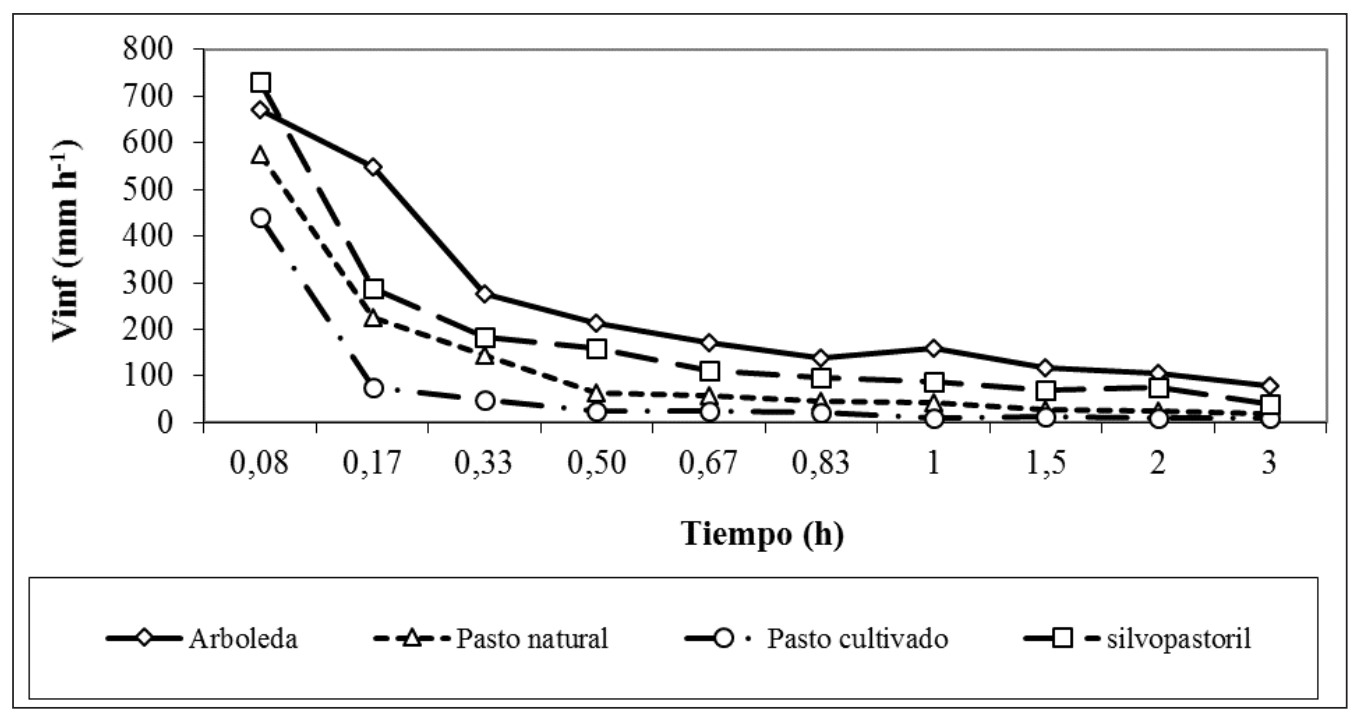

Figura 2. Velocidad de infiltración básica $\left(\mathrm{mm} \mathrm{h}^{-1}\right)$ de los diferentes sistemas de uso del suelo en las Tunas, Cuba. 
Leyva et al. (2012), en estudios de la macrofauna del suelo en estos ecosistemas, encontraron valores superiores de densidad y biomasa de oligoquetos y mayor diversidad de otros individuos de la macrofauna en los sistemas silvopastoriles y arboleda, demostrando que la presencia de árboles en los pastizales de gramínea potencian y diversifican las comunidades de macroinvertebrados del suelo, resultados que coinciden con estudios realizados por Camero-Rey y Rodríguez-Díaz (2015) al encontrar mayor número de lombrices en el sistema silvopastoril con respecto a los monocultivos de pastos.

Materia orgánica. El mayor contenido de materia orgánica en el suelo, se encuentra en los primeros $5 \mathrm{~cm}$ de profundidad, con los niveles más altos para el sistema silvopastoril; los demás sistemas presentan niveles medios (Tabla 4). Resultados similares obtuvieron Cairo-Cairo et al. (2017) con incrementos del contenido de materia orgánica en un bosque introducido, que funcionó como un sistema silvopastoril, debido no solo al aporte de la hojarasca, sino también a la incorporación de estiércol de bovinos y caprinos. Estudios realizados por Murray et al. (2014), a ocho años de implantado el sistema agroforestal mostró un incremento del 85\% de la materia orgánica edáfica en el horizonte $\mathrm{A}$.

Tabla 4. Contenidos de materia orgánica (\%) en los diferentes sistemas de uso del suelo en las Tunas, Cuba.

\begin{tabular}{lcccc}
\hline \multirow{2}{*}{\multicolumn{1}{c}{ Sistemas }} & \multicolumn{4}{c}{ Profundidad (cm) } \\
\cline { 2 - 5 } & $0-5$ & $5-20$ & $20-30$ & $30-40$ \\
\hline Arboleda & $4,90 \mathrm{a}$ & $3,47 \mathrm{~b}$ & $2,80 \mathrm{~b}$ & $1,97 \mathrm{~b}$ \\
Pastos naturales & $4,71 \mathrm{a}$ & $3,10 \mathrm{~b}$ & $1,78 \mathrm{c}$ & $1,57 \mathrm{c}$ \\
Pastos cultivados & $4,75 \mathrm{a}$ & $4,66 \mathrm{a}$ & $4,24 \mathrm{a}$ & $3,04 \mathrm{a}$ \\
Silvopastoril & $5,20 \mathrm{a}$ & $3,30 \mathrm{~b}$ & $2,61 \mathrm{~b}$ & $2,15 \mathrm{~b}$ \\
Error estándar & 0,10 & 0,15 & 0,21 & 0,13 \\
\hline
\end{tabular}

Letras distintas en cada columna indican diferencias significativas $(\mathrm{p}<0,05)$.
Los valores más altos en profundidad se obtuvieron en el pasto cultivado con diferencias respecto a los demás sistemas. En los horizontes inferiores en el sistema de pasto cultivado, los contenidos de materia orgánica disminuyen en un 10 y 30\% respecto a los primeros $5 \mathrm{~cm}$, en los demás sistemas, la diferencia oscila entre 35 y $65 \%$.

El mayor contenido de materia orgánica en los pastos cultivados, podría atribuirse a los altos contenidos de residuos, principalmente de $P$. purpureum y C. nlemfuensis sobre la superficie del suelo. Según Moussadek et al. (2014), la retención de los residuos de gramíneas en la superficie del suelo, aumenta el contenido de carbono orgánico y de los agregados estables en agua.

Los residuos de ambas gramíneas presentan altos contenidos de lignina, con una alta relación carbono/nitrógeno. De acuerdo con Orellana et al. (2008), permiten una lenta mineralización y enriquecimiento de la materia orgánica. Lo anterior, confirma que los flujos de $\mathrm{C}$ del suelo dependen directamente de los de $\mathrm{N}$, debido a que las relaciones C:N altas limitan la descomposición de materiales orgánicos.

En el sistema silvopastoril, los menores contenidos de materia orgánica en profundidad, están dados por la presencia de materiales orgánicos de menor relación $\mathrm{C}: \mathrm{N}$, debido al aporte del nitrógeno mediante la asociación simbiótica de las leguminosas con bacterias del género Rhizobium (Bueno y Camargo, 2015) y a la biomasa que es rápidamente ciclada por los organismos del suelo (Prager et al., 2012). Huamancayo y Robles (2014) reportan en sistemas de pastos, mayores reservas de materia orgánica que en sistemas silvopatoriles en los dos últimos niveles de profundidad. Según Seddaiu et al. (2013), la acumulación del carbono orgánico en el horizonte muy superficial de los suelos bajo bosques y la disminución en el horizonte subyacente puede limitar la capacidad resistente de éstos a 
factores de perturbación, mientras que los suelos de las praderas puede ser considerado más resistente por el mayor secuestro de carbono en un horizonte de mayor espesor.

A pesar de los altos contenidos de carbono en el sistema pasto cultivado, su relación con otros indicadores y funciones del suelo no fue directa. En este caso, la cantidad no estuvo asociada al mejor estado del sistema, dado fundamentalmente por el manejo. Según Janzen et al. (1992), la relación entre los diferentes indicadores y las funciones del suelo no siempre es directa y puede no necesariamente estar asociada la cantidad con el mejor estado del sistema. Estudios realizados por Lok et al. (2009), en bancos de biomasa de CT-115, demostraron que siempre que sea adecuadamente manejado el suelo, éste conserva y mejora su fertilidad, aunque con el tiempo de explotación, la resistencia a la penetración aumenta.

Actividad de la enzima $\boldsymbol{\beta}$-glucosidasa. La mayor actividad de la enzima $\beta$-glucosidasa en la profundidad de $0-10 \mathrm{~cm}$ (Tabla 5), se encontró en el sistema silvopastoril, con diferencias significativas respecto de los demás sistemas. Esto corrobora el mejor funcionamiento del suelo en este sistema, que propicia condiciones adecuadas para la actividad microbiana, dado por la calidad y diversidad de los residuos orgánicos en estos sistemas. Navas (2008) encontró mayor actividad de la $\beta$-glucosidasa en suelos con cobertura de C. rotundifolium, por tener esta especie la particularidad que se defolia completamente en la época seca, y a una mayor velocidad de descomposición en el tiempo.

Entre los sistemas de pastos cultivados y naturales, no existen diferencias significativas, pero la actividad de la enzima es menor, posiblemente por condiciones inadecuadas de aireación para la actividad microbiana y composición de residuos más lignificados (gramíneas). León (2010) encontró menor actividad de la enzima ß-glucosidasa con la incorporación de residuos de alta relación C:N. Zacheis et al. (2002), plantean que el ganado puede ofrecer un incremento de la actividad enzimática por el suministro extra de materia orgánica en el suelo, pero puede provocar un detrimento asociado a la perturbación de la estructura del suelo (Cao et al., 2004).

En la profundidad de $10-20 \mathrm{~cm}$ en todos los sistemas, excepto en el pasto cultivado, la actividad enzimática, disminuyó con respecto al estrato superior. Los mayores valores se obtuvieron en la arboleda, seguido del silvopastoril. El pasto natural, alcanzó el menor valor, sin embargo, el pasto cultivado incrementó su actividad a esta profundidad. Esto se relaciona con el alto contenido de materia orgánica de este sistema, que a esta profundidad, está más degradada y accesible a los microorganismos. Con presencia de gran número de raíces, que en el pasto natural, se encuentran sobre todo en el estrato superficial. Ambos sistemas no alcanzan valores superiores por su alta compactación y pobre drenaje. Los valores encontrados para esta enzima, concuerdan con los reportados por Henríquez et al. (2014), bajo diferentes manejos y tipos de suelos.

Tabla 5. Actividad de la enzima $\beta$-glucosidasa en los diferentes sistemas de uso del suelo en la Tunas Cuba.

\begin{tabular}{lcc}
\hline \multirow{1}{*}{ Sistemas } & \multicolumn{2}{c}{ Profundidad (cm) } \\
\cline { 2 - 3 } & $\mathbf{0 - 1 0}$ & $\mathbf{1 0 - 2 0}$ \\
\hline Arboleda & $157,4 \mathrm{~b}$ & $148,0 \mathrm{a}$ \\
Pasto natural & $107,4 \mathrm{c}$ & $49,3 \mathrm{~d}$ \\
Pasto cultivado & $112,5 \mathrm{c}$ & $121,0 \mathrm{c}$ \\
Silvopastoril & $200,1 \mathrm{a}$ & $128,34 \mathrm{~b}$ \\
Error estándar & 11,38 & 11,25 \\
\hline
\end{tabular}

Letras distintas en cada columna indican diferencias significativas $(\mathrm{p}<0,05)$. 


\section{CONCLUSIONES}

Las propiedades estudiadas demostraron ser sensibles a las variaciones en el tiempo, en dependencia del sistema de uso y manejo. El incremento de la densidad aparente influyó sobre la mayoría de las propiedades del suelo, causa principal de los bajos valores de infiltración y porosidad, especialmente en los sistemas de pastos naturales y cultivados.

En el sistema silvopastoril, se encontró la mayor actividad de la enzima $\beta$-glucosidasa, menor densidad aparente, mayor velocidad de infiltración, porosidad y materia orgánica en superficie lo que constituye una alternativa para la recuperación de los suelos de pastizales en los sistemas ganaderos de la región.

Conflicto de intereses: Los autores declaran que no hay conflicto de interés.

\section{REFERENCIAS BIBLIOGRÁFICAS}

Bueno, L. \& Camargo, J.C. (2015). Nitrógeno edáfico y nodulación de Leucaena leucocephala (Lam.) de Wit en sistemas silvopastoriles. Acta Agron. 64 (4): 349-354. doi: http://dx.doi.org/10.15446/acag. v64n4.45362.

Cairo-Cairo, P., Reyes-Hernández, A., Aro-Flores' R.V. \& Robledo-Ortega, L. (2017). Efecto de las coberturas en algunas propiedades del suelo. Finca La Morrocoya, Barinas, Venezuela. Pastos y Forrajes. 40 (2): 127-134.

Camero-Rey, A. \& Rodríguez-Díaz, H. (2015). Características químicas del suelo, producción forrajera y densidad poblacional de lombrices en un sistema silvopastoril en la zona Huetar Norte de Costa Rica. Tecnología en Marcha. 28(1): 91-104.

Cao, G., Tang, Y., Mo, W., Wang, Y., Li, Y. \& Zhao, X. (2004). Grazing intensity alters soil respiration in an alpine meadow on the Tibetan plateau. Soil Biology \& Biochemistry. 36: 237-243.
Celik, A. \& Raper R.L. (2012). Design and evaluation of ground-driven rotary subsoilers. Soil \& Tillage Research. 124: 203-210. doi: 10.1016/j. still.2012.06.010

Chará, J., Camargo, J. C., Calle, Z., Bueno, L., Murgueitio, E., Arias, L., Dossman, M. A. \& Molina, E. J. (2015). Servicios ambientales de sistemas silvopastoriles intensivos: mejoramiento del suelo y restauración ecológica. pp. 331-344. En: Montagnini, F., Somarriba, E., Murgueitio, E., Fassola, H., Eibl, B. Sistemas Agroforestales. Funciones productiva, socioeconómica y ambiental. Serie técnica. Informe técnico 402. CATIE. Turrialba, Costa Rica: CIPAV. 454p.

Chavarría, N., Tapia, A. C., Soto, G. \& Filho, M.V. (2012). Efecto de diferentes sistemas de manejo sobre la calidad del suelo, en fincas cafetaleras de la zona de Turrialba y Orosi. InterSedes: Revista de las Sedes Regionales. XIII (26): 83-105.

García; L.M., Mesa, A.R. \& Hernández, M. (2014). Potencial forrajero de cuatro cultivares de Pennisetum purpureum en un suelo Pardo de Las Tunas. Pastos y Forrajes. 37(4): 413-419.

Gaspar, L. \& Navas, A. (2013). Vertical and lateral distributions of 137Cs in cultivated and uncultivated soils on Mediterranean hillslopes. Geoderma. 207-208: 131 - 143. doi: 10.1016/j.geoderma.2013.04.034.

Gómez, G.C., Munive, R., Mallma, T. \& Orihuela, C. (2014). Evaluación de la tasa de infiltración en tierras agrícolas, forestales y de pastoreo en la subcuenca del río Shullcas. Apuntes ciencias sociales. 04(01): 32 - 43.

Griffith, D.R., Mannering, J.V. \& Moldenhauer, W.C. (1977). Conservation tillage in eastern Corn Belt. J. Soil Water Conserv. 32(1): 20-28.

Henríquez, C., Uribe, L., Valenciano, A. \& Nogales, R. (2014). Actividad enzimática del suelo-deshidrogenasa, $\beta$-glucosidasa, fosfatasa y ureasa- bajo diferentes cultivos. Agronomía costarricense. 38 (1): 43-54.

Hernández, A., Morell, F., Ascanio, M.O., Borges, Y., Morales, M. \& Yong, A. (2006). Cambios Globales de los suelos Ferralíticos rojos lixiviados (Nitisoles ródicos éutricos) de la provincia La Habana. Cultivos Tropicales. 27 (2): 41-50. 
Hernández, A., Paneque, J., Pérez, J. M. \& Fuentes, E. (1995). Manual para la cartografía detallada y evaluación integral de los suelos. La Habana: Instituto de Suelos. 52p.

Hoffmann, G. \& Dedeken, M. (1965). Eine methode zurbvkolori metrschen Bestimmung der ß- lucosidase aktivitat in boden. Pflanzenernachr Bodenkd. 108:195-201.

Huamancayo, G.G. \& Robles, R.R. (2014). Carbono almacenado en tres sistemas ganaderos en el distrito de José Crespo y Castillo, Aucayacu. pp. 24-42. En: resúmenes de artículos científicos de trabajos de investigación realizados en la unidad familiar de producción sostenible en el trópico húmedo - Aucayacu. Perú: Universidad Nacional Agraria de la Selva.

IUSS Grupo De Trabajo WRB. (2007). Base Referencial Mundial del Recurso Suelo. Primera actualización 2007. Informes sobre Recursos Mundiales de Suelos No. 103. Roma: FAO. 117p.

Janzen, H. H., Larney, F. J. \& Olson, B. M. (1992). Soil quality factors of problem soils in Alberta. Proceedings of the Alberta Soil Science Workshop. 29:17-28.

Kassam, A. (2014). Save and Grow: Soil health. Paper presented at the FAO Technical Consultation on Save and Grow: Maize, Rice and Wheat. En: https:// kupdf.com/download/9-fao-save-and-grow-inpractice-maize-rice-wheatpdf_5a397356e2b6f58d 08d45b4d_pdf.

León, P. (2010). Evaluación de la actividad enzimática en suelos enmendados bajo diferentes formas de uso y manejo. España, Madrid: Universidad Politécnica de Madrid.

Leyva, L., Baldoquin, A., Ruz, R. \& Ayala J.R. (2012). Influencia del uso del suelo en la macrofauna edáfica en áreas de la región norte del municipio de Las Tunas. Revista de innovación tecnológica de Las Tunas. 18 (2): 13-26.

Leyva, S. L., Masaguer, A. \& Baldoquin, A. (2014). Efectos de sistemas de labranza en Luvisoles dedicados a la producción de pastos. Pastos y forrajes. 37(4): 408-412.

Lok, S. (2016). Soils dedicated to cattle rearing in Cuba: characteristics, management, opportunities and challenges. Cuban Journal of Agricultural Science. 50(2): 279-290.
Lok, S., Crespo, G., Torres V., Fraga, S. \& Nod A. (2009). Impacto de la tecnología de banco de biomasa de Pennisetum purpureum CUBA CT-115 en el sistema suelo-pasto-animal de una unidad de producción de leche con ganado vacuno. Revista Cubana de Ciencia Agrícola. 43(3): 307.

Lok, S., Fraga, S., Noda, A. \& García, M. (2013). Almacenamiento de carbono en el suelo de tres sistemas ganaderos tropicales en explotación con ganado vacuno. Revista Cubana de Ciencia Agrícola. 47 (1): 75-82.

Lozano, P.Z., Lobo, L.D. \& Pla, S. I. (2000). Diagnóstico de limitaciones físicas en Inceptisoles de los llanos occidentales venezolanos. Bioagro. 12 (1): 15-24.

Montagnini, F., Somarriba, E., Murgueitio, E., Fassola, H. \& Eibl, B. (2015). Sistemas Agroforestales. Funciones Productivas, Socioeconómicas y Ambientales. Serie técnica. Informe técnico 402. CATIE, Turrialba. Costa Rica: Editorial CIPAV. 454p.

Moussadek, R., Mrabet, R., Dahan, A., Zouahri, M., Mourid, E. \& Van Ranst, E. (2014). Tillage System Affects Soil Organic Carbon Storage and Quality in Central Morocco. Applied and Environmental Soil Science. Article ID 654796 doi:10.1155/2014/654796.

Munkholm, L.J. (2011). Soil friability: A review of the concept, assessment and effects of soil properties and management. Geoderma. 167-168: 236-246. doi:10.1016/j.geoderma.2011.08.005.

Murgueitio, E., Barahona, R., Xochilt F., Chará, J.D. \& Rivera J.E. (2016). Es Posible Enfrentar el Cambio Climático y Producir más Leche y Carne con Sistemas Silvopastoriles Intensivos. Ceiba. 54(1): 23-30. doi: 10.5377/ceiba.v54i1.2774.

Murillo, J., Rodríguez, G., Roncallo, B., Rojas, L.A. \& Bonilla, R.R. (2014). Efecto de la aplicación de prácticas sostenibles en las características físicas, químicas y microbiológicas de suelos degradados. Pastos y Forrajes. 37 (3): 270-278.

Murray, R. M., Orozco, M. G., Hernández, A., Lemus, C. \& Nájera, 0. (2014). El sistema agroforestal modifica el contenido de materia orgánica y las propiedades físicas del suelo. Avances en Investigación Agropecuaria AIA. 18(1): 23-31.

Navas, M. (2008). Sistemas de producción "Ley Farming". Caracterización, evaluación y su efecto sobre las propiedades de un suelo en los llanos orientales de Venezuela. Madrid: Universidad Politécnica de Madrid. 
NC ISO 10272. (2003). Norma Cubana. Calidad del Suelo. Determinación de la Densidad Aparente o peso volumétrico. Ciudad de la Habana, Cuba: Oficina Nacional de Normalización.

NC - Norma Cubana. (2010). NC: 20. Calidad del suelo. Determinación de la porosidad y capacidad de campo. Ciudad de la Habana, Cuba: Oficina Nacional de Normalización.

NRAG 408. (1981). Norma Ramal. Suelos. Análisis mecánico. Determinación. Oficina Nacional de Normalización. Ciudad de la Habana, Cuba: NRAG.

NRAG-128. (2009). Norma Ramal. Calidad del Suelo. Determinación de la densidad de las partículas. Oficina Nacional de Normalización. Ciudad de la Habana, Cuba: NRAG.

Orellana, G. R., Ortega S.F. \& Moreno, A.J.M. (2008). Fracción orgánica ligera del suelo como indicador agroecológico. Revista de Agricultura Orgánica. 2: 40-41

Porta, J., López-Acevedo, M. \& Roquero De Laburu, C. (2003). Edafología. Para la agricultura y el medio ambiente.3ra. Madrid, España: Mundi-Prensa. 929 p.

Prager, M., Sanclemente, 0., Sánchez De Prager, M., Gallego, J. \& Ángel, D. (2012). Abonos verdes: tecnología para el manejo agroecológico de los cultivos. Agroecología. 7: 53-62.

Raper, R. L. (2005). Force requirements and soil disruption of straight and bentleg subsoilers for conservation tillage systems. Appl. Eng. Agric. 21 (5): 787-794.

Sadeghian, S., Rivera, J.M. \& Gómez, M. E. (2010). Impacto de sistemas de ganadería sobre las características físicas, químicas y biológicas de suelos en los Andes de Colombia. Conferencia electrónica de la FAO sobre "Agroforestería para la producción animal en Latinoamérica". Recuperado de http://www. fao.org/livestock/agap/frg/agrofor1/Siavosh6.htm

Seddaiu, G., Porcua, G., Leddaa, L., Roggeroa, P.P., Agnellib A. \& Cortic G. (2013). Soil organic matter content and composition as influenced by soil management in a semi-arid Mediterranean agro-silvo-pastoral system. Agriculture. Ecosystems and Environment. 167: 1-11.
Silva, G.L., Lima H.V., Campanha, M.M., Gilkes, R.J. \& Oliveira, T.S. (2011). Soil physical quality of Luvisols under agroforestry, natural vegetation and conventional crop management systems in the Brazilian semi-arid region. Geoderma. 167-168: 61-70. doi:10.1016/j.geoderma.2011.09.009.

Strobl, W. \& Traunmüller, M. (1996). B-Glucosidase Activity. pp.198-200. In: Schinner, F., Öhlinger, R., Kandeler, E., Margesin, R. (Ed.). Methods in Soil Biology. Germany: Springer.416p.

Vadiunina, A.F. \& Korchaguina, Z.A. (1986). Métodos de desarrollo de las propiedades físicas del suelo. Tercera edición. Moscú: MIR. 416p.

Verhulst, N., François, I. \& Govaerts, B. (2015). Agricultura de conservación y captura de carbono en el suelo: Entre el mito y la realidad del agricultor. México, D.F.: CIMMYT. 16p.

Walkley, A. \& Black, T. A. (1934). An examination of the Degtjareff method for determining soil organic matter and a proposed modification of the chromic acid titration method. Soil Sci. 63: 251-264.

Zacheis, A., Ruess R.W. \& Hupp J.W. (2002). Nitrogen dynamics in an Alaskan salt marsh following spring use by geese. Oecologia. 130: 600-608. 